\title{
RESPONSABILIDAD SOCIAL CORPORATIVA EN CADENAS HOTELERAS: HOTEL LAS AMÉRICAS EN CARTAGENA DE INDIAS
}

\author{
Alain Castro-Alfaro \\ Lino Mercado-León \\ Nora González-Pérez
}


Panorama Económico, 24 (Octubre 2016 - Septiembre 2017), pp. 283-296

Alain Castro-Alfaro

Lino Mercado-León

Nora González-Pérez

\title{
Responsabilidad social corporativa en cadenas hoteleras: Hotel las Américas en Cartagena de Indias
}

\begin{abstract}
Resumen
La responsabilidad social corporativa o empresarial (RSE), es la práctica empresarial donde se manifiesta el buen gobierno que emana desde la alta dirección y que se es visible por medio del equilibrio entre los fines económicos de la organización y el impacto que estas producen en el entorno social donde se llevan a cabo las actividades. El presente artículo de investigación tiene como objetivo principal el determinar la manera en que se está llevando a cabo las políticas de RSE en el hotel Las Américas en la ciudad de Cartagena de Indias. Se identificó el accionar que tiene el hotel en relación a sus grupos de interés o stakeholders. La metodología utilizada fue del tipo descriptivo y como método fue el de la observación directa. Como fuente de recolección de información primaria se tuvo una encuesta dirigida al gerente del hotel. Como conclusión, se puede decir que el hotel está ampliamente comprometida con el tema de la responsabilidad social dentro de su gestión corporativa.
\end{abstract}

Palabras Clave: Responsabilidad social empresarial, hoteles, grupos de interés, dimensiones.

Clasificación JEL: M14, I31, D11.

\section{Corporate social responsibility in hotel chains: \\ The case of the Las Americas Hotel in Cartagena de Indias}

\begin{abstract}
"Corporate social responsibility (CSR) is the business practice where good governance emanates from top management and is visible through a balance between the economic goals of the organization and the impact they produce in the organization. Social environment where activities are carried out. This paper is a product of the research group Cartaciencias, which is recognized by Colciencias and, in turn, is part of the public accounting program of the Rafael Nuñez University Corporation. This research article has as main objective to determine the way in which the policies of CSR are being carried out in the Hotel Las Américas in the city of Cartagena de Indias. It was identified the action that the hotel has, through a survey directed company manager, before its stakeholders or stakeholders. The methodology used was descriptive, hand in hand with direct observation. As a source of primary information collection, a survey was conducted focusing on the hotel manager where the dimensions in the study of CSR are taken into account. As a conclusion, it can be said that the hotel is widely committed to the issue of social responsibility within its corporate management. Keywords: Corporative social responsibility, hotel,, stakeholders, dimensions.
\end{abstract}

JEL Classification: M14, I31, D11.

\section{Responsabilité sociale de l'entreprise dans les chaînes: Le cas Des Amériques Hôtel Cartagena}

\section{Résumé}

La responsabilité sociale des entreprises ou RSE est une expression de bonne gouvernance venant de la haute direction, perçue comme un équilibre entre le gain corporatif et l'impact social dans le secteur avoisinant. Cet article analyse les politiques RSE utilisées par Hotel Las Americas de Carthagène des Indes en identifiant les principales parties prenantes. La méthodologie est descriptive de type observation directe. La source première d'information est un sondage dirigée au gestionnaire principal de l'hôtel. Ces informations permettent de conclure que l'hôtel est grandement impliqué avec la communauté et prends à cœur sa responsabilité sociale.

Mots-clés: Responsabilité sociale des entreprises, hôtellerie, dimensions, parties prenantes.

Nomenclature JEL: M14, I31, D11. 


\section{RESPONSABILIDAD SOCIAL CORPORATIVA EN CADENAS HOTELERAS: HOTEL LAS AMÉRICAS EN CARTAGENA DE INDIAS}

INFORMACIÓN DEL ARTÍCULO Recepción de articulo: 14 de mayo de 2016 Aceptación de artículo: 1 de septiembre de 2016

\author{
Alain Castro-Alfaro ${ }^{1}$ \\ Corporación Universitaria Rafael Núñez \\ Colombia \\ Lino Mercado-León \\ Fundación Universitaria Tecnológico Comfenalco \\ Colombia \\ Nora González-Pérez \\ Universidad IAFIT \\ Colombia
}

\section{INTRODUCCIÓN}

Hablar de globalización, la cual es analizada desde diversos ángulos, y que tiene repercusiones en las sociedades, es conceptualizado por diversos autores e instituciones. Citando al Fondo Monetario Internacional (Castro, 2016) enuncia que el proceso globalitario se da como la interdependencia económica cada vez más progresiva entre las naciones del mundo, dándose como consecuencia el incremento de las transacciones de bienes y capitales, a su vez impulsado por el constante avance tecnológico. Debido a ello, Anturi (2015), expone que el ambiente sufre como resultado del incremento productivo y comercial, lo cual se traduce en el vertedero de desechos en la naturaleza. Por otro lado, no hay que perder de vista que el proceso global se manifiesta de diferentes formas entre los países (Castro, 2013), siendo que unos logran aprovechar tal coyuntura para lograr desarrollarse más y, en cambio, los que al no poder ser competitivos, experimentan retroceso. Por lo anterior, "toda organización debe aspirar a la transformación constante en pro de un mejor rendimiento" (Del Río, Cardona, Pérez, 2012).

En palabras de Ruiz y Romero (2011), La RSE se presenta como "un proceso que deberedundar en una práctica empresarial que satisfaga preocupaciones sociales y medioambientales con una dirección interior-exterior y donde intervienen sujetos activos y pasivos" (p.128), lo cual quiere decir que la organización como sujeto activo, tiene en cuenta e informa a su público interno y entorno como sujetos pasivos, de tal forma que estos últimos puedan evaluar los esfuerzos en pro de la sociedad que lleva a cabo el ente corporativo.

${ }^{1}$ Autor para correspondencia. Correo electrónico: alain.castro@curnvirtual.edu.co 


\section{INTRODUCCIÓN}

Hablar de globalización, la cual es analizada desde diversos ángulos, y que tiene repercusiones en las sociedades, es conceptualizado por diversos autores e instituciones. Citando al Fondo Monetario Internacional (Castro, 2016) enuncia que el proceso globalitario se da como la interdependencia económica cada vez más progresiva entre las naciones del mundo, dándose como consecuencia el incremento de las transacciones de bienes y capitales, a su vez impulsado por el constante avance tecnológico. Debido a ello, Anturi (2015), expone que el ambiente sufre como resultado del incremento productivo y comercial, lo cual se traduce en el vertedero de desechos en la naturaleza. Por otro lado, no hay que perder de vista que el proceso global se manifiesta de diferentes formas entre los países (Castro, 2013), siendo que unos logran aprovechar tal coyuntura para lograr desarrollarse más y, en cambio, los que al no poder ser competitivos, experimentan retroceso. Por lo anterior, "toda organización debe aspirar a la transformación constante en pro de un mejor rendimiento" (Del Río, Cardona, Pérez, 2012).

En palabras de Ruiz y Romero (2011), La RSE se presenta como "un proceso que debe redundar en una práctica empresarial que satisfaga preocupaciones sociales y medioambientales con una dirección interior-exterior y donde intervienen sujetos activos y pasivos" (p. 128), lo cual quiere decir que la organización como sujeto activo, tiene en cuenta e informa a su público interno y entorno como sujetos pasivos, de tal forma que estos últimos puedan evaluar los esfuerzos en pro de la sociedad que lleva a cabo el ente corporativo.
El punto principal que tiene la RSE se enfoca en la voluntariedad por lo socialmente responsable, por medio de ello se conseguirá aumentar los efectos positivos en la humanidad (Bateman y Snell, 2005) y se logrará disminuir los negativos efectos, trayendo como consecuencia en beneficios en el desarrollo sostenible y a largo plazo. De igual manera, Serna, Barrera y Montiel (2011), exponen que por medio de la sustentabilidad, las organizaciones y los Estados deben de analizar los resultados económicos, ambientales y sociales, con el fin de implementar estrategias que permitan generar nuevas oportunidades de negocio pero con alto sentido de respeto al medio ambiente. Para que ello se pueda poner en práctica, Castro (2014), expresa que los entes corporativos deben de ser cumplidoras de la normatividad ambiental, ya que de esa manera se podrán ofrecer productos y servicios de calidad y respetuosas del medio ambiente. Consecuentemente, las empresas deben de comprender el entorno donde llevan a cabo sus operaciones, de la mano de una clara comprensión de su internalidad, es decir las reglas y leyes que presiden sus operaciones.

Existen grupos que conforman el estudio de la RSE en los entes corporativos, estos son los llamados grupos de interés o stakeholders, siendo aquellos compuestos por el público interno y externos, ante los cuales la empresa tiene sus acciones y ejerce su influencia.

Por lo anteriormente dicho, esta investigación tuvo como objetivo central determinar el estado de la responsabilidad social corporativa en el hotel cinco estrellas Las Américas.

\section{MARCO REFERENCIAL}

La RSE se sustenta en principios y valores y no de títulos valores, estos permiten un 
comportamiento socialmente responsable que incluyen todos los intereses que son lícitos en el accionar corporativo y, aunado a las respuestas que debe de dar el ente ante las nuevas demandas sociales que no necesariamente están regentadas en las leyes de un Estado (Almagro, 2009).

A su vez, se destaca el concepto que da la Comisión Europea (citado en Server y Capó, 2011), en la cual expresa que la RSE se presenta cuando las organizaciones logran la integración de los aspectos sociales y ambientales en su ejercicio cotidiano por medio de sus procederes $y$ su manera de interrelacionarse con sus grupos de interés.

El Instituto Ethos de Empresas y Responsabilidad social y el Servicio Brasileño de apoyo a la Micro y Pequeñas Empresas "SEBRAE" (citado en Vergara y Carbal, 2014), la responsabilidad social de la empresa en una forma gestionaría que se caracteriza por la transparencia y la ética en todas las actividades del ente corporativo con los públicos a los cuales tiene contacto y que sus metas sean respetuosas con el desarrollo sostenible, con el fin de preservar los recursos naturales para las generaciones venideras. A su vez, Castro (2013), enfatiza la importancia de que las empresas tengan el enfoque ético en sus operaciones sin dejar de lado la generación de utilidades económicas.

Ante lo anteriormente expresado, se puede interpretar que la RSE tiene en su seno dos aspectos que le son fundamentales: en primer lugar, una visión que fomenta la manera en la cual deben de comportarse los empresarios para con la sociedad y, en segundo lugar, la apelación al cambio de conducta empresarial, donde se incluya en su gestión los temas sociales y medioambientales aunque la ley no lo exija necesariamente.
ICONTEC en el año 2008, por medio de la GTC 180 de la responsabilidad social dice que la RSE es un compromiso potestativo que las organizaciones toman en relación a los intereses que tienen para con sus grupos de interés, lo cual generará desarrollo económico pero respetando el equilibrio socio ambiental. Por ello, citando a Navas y Londoño (2015), la RSE parte del importante papel que el ente corporativo desempeña en la realidad social y del considerable impacto de su accionar.

La responsabilidad social denota una acción consciente, la cual emerge de la parte volitiva y cuya base se funda en reconocer el compromiso que se debe tener para con la sociedad. A su vez, es proactiva dando como resultado la iniciativa de proceder teniendo presente la disminución de los impactos negativos hacia el medio ambiente y la sociedad en general. Esta se puede presentar de manera individual o colectiva, tal el caso de organizaciones empresariales. Como ejemplo, se cita a Ocampo (2014), donde expone las acciones del banco Davivienda, donde por medio de su programa "Mi finanza en casa", tiene como fin brindar información básica concerniente al manejo de las finanzas que se manejan en la cotidianidad desde la óptima personal y dirigida tanto a niños como adultos.

La RSE está teniendo un avance importante en las últimas décadas, debido a la importancia que está tomando en naciones desarrolladas y, esta repercusión, está llegando a América Latina donde ya existen empresas que dentro de su gestión han implantado las prácticas socialmente responsables. De esta manera, gracias al acelerado comercio, el crecimiento poblacional y el uso de los recursos naturales a grandes escalas, las empresas tienen mayores responsabilidades que 
solamente la mera ganancia, sino también de cuidar la naturaleza y que esta sea sostenible, tal como lo expresan Vives y Peinado-Vara (2011).

Por lo tanto, en un mercado mundial cada vez más competitivo, ágil y agresivo (Cifuentes, 2012; Quesada, 2003; Vergara et al., 2008), la RSE se puede convertir en una ventaja competitiva y pueda diferenciarse de los de la competencia, logrando su permanencia y con resultados amigables con el entorno donde lleva a cabo sus actividades (Londoño, 2007; Maldonado, 2015).

Sin lugar a dudas, uno de los aspectos que son más evidentes en la actualidad es que las organizaciones han percibido que el cuidado del medio ambiente no son elementos adicionales, ni fuera del ámbito de la empresa sino que son imprescindibles para darle la debida sustentabilidad económica. Sin embargo, para llevar adelante esta práctica a mayor escala hay mucho camino por andar. Cortés (2013), expresa que "además de la ética empresarial e individual y la concientización de los empresarios, es necesario el desarrollo del mercado de la responsabilidad, donde las partes interesadas puedan hacer valer sus opiniones y donde los gobiernos ejerzan eficientemente su papel regulador (p. 16).

La función empresarial en la sociedad va más lejos que la mera producción de bienes y servicios, de la generación de empleos así como del cumplimiento de las normatividades legales. Hay que tener presente que los individuos son seres sociales y se necesitan entre sí para poder desarrollarse, además de que las empresas son gestionadas por personas (Castro, Marrugo, Gutiérrez y Camacho, 2014). Por lo dicho, los entes corporativos necesitan de seres humanos para poder llevar adelante su actividad y, si se respeta el entorno social, obtendrán la debida recompensa de ella.

Mitchell, Agle y Woods (1997), dieron una propuesta de identificación sobre los stakeholders la cual permite darle operacionalidad al concepto teniendo como base en su énfasis o preponderancia, en relación de la presencia simultánea de determinados atributos en actores que afectan y son afectados por los objetivos de un ente corporativo. Tales actores son internos y externos que son afectados por el accionar empresarial en diferentes niveles, en la medida en que tienen entre uno $y$ tres atributos fundamentales que son: en primer lugar, poder, lo cual significa que un stakeholder puede tener la posibilidad de obtener recursos para lograr imponer su deseo ante otros; en segundo lugar, legitimidad, la cual es la percepción apropiada que se tiene del accionar de un actor los cuales se encuentra dentro de un sistema de valores y normas; por último, urgencia, la cual expone por el deseo de una atención inmediata.

Los stakeholders o también llamados grupos de interés, según Castro, Hernández y Bedoya (2016), son aquellos que son afectados o tienen intervención directa o indirecta en el proceso de las operaciones del ente corporativo. A su vez, Freeman (1984), fue el que dio a conocer esta palabra dentro del concepto de la RSE. Por medio de esta aseveración, la empresa debe tener un compromiso moral con los individuos o grupos que, tanto internos como externos, tengan relación con la organización. Estos stakeholders están compuestos por los empleados, clientes, proveedores, accionistas, gobierno u otros. Por lo anteriormente expuesto, se puede decir que una entidad no puede estar independiente de sus grupos de interés, sino más bien se encuentran integrados dentro de la misma. 
La Confederación de la Producción y del Comercio y Prohumana (2006), presenta seis dimensiones de la RSE las cuales son tomados como referencias para el análisis del hotel Las Américas. Estas son: a) Valores y coherencia, la cual está referenciada al comportamiento ético de un ente corporativo. A su vez, tiene cinco subdimensiones que son: Código de ética, Gobierno corporativo, Transparencia en informes contables, Relaciones leales con la competencia y Reportes de sustentabilidad; b) Público interno, donde se observa la práctica corporativa relacionada con los empleados. Esta tiene subdimensiones, que son: Seguridad e higiene, Política de salud, Políticas con los sindicatos, Políticas de contratación, Políticas de capacitación, Políticas de beneficios, Conciliación de vida personal y vida laboral, Política de previsión, Políticas de prevención y sanción del acoso y Política de Diversidad; c) Relación con los proveedores, donde se enfatiza en la accionar de la empresa en relación a los que lo abastecen. De igual forma, tiene subdimensiones, a saber: Desarrollo de proveedores, Encadenamiento productivo, Aspectos laborales y Subcontratación; d) Relación con los consumidores, siendo este de vital importancia para el desarrollo de la empresa, esto porque según como es su comportamiento se verá reflejado en el aumento, o por el contrario, en la disminución de sus ventas. Asimismo, tiene sus subdimensiones, que son: Satisfacción de los consumidores, Política publicitaria y Conocimiento de daños potenciales; e) Relación con la comunidad, donde se puede observar en la manera como el ente corporativo se relaciona con su entorno cercano y con la sociedad en donde está inserta. Las subdimensiones son: Diálogos con Públicos de Interés, Vinculación Comunidad/ Inversión Social y Voluntariado Corporativo; y f) Medioambiente, siendo aquí donde se puede analizar la relación que tienen las actuaciones de la organización para con el impacto medioambiental. Esta tiene las siguientes subdimensiones: Actitud proactiva frente a exigencias medioambientales, Prácticas ambientales y Sistema de gestión ambiental.

\section{METODOLOGÍA}

La investigación está enmarcada dentro del enfoque cuantitativo y es a su vez del tipo descriptivo. De igual forma, se tomó el diseño no experimental.

Las técnicas que se utilizaron en el levantamiento de la información fueron la observación y una encuesta a la gerencia del hotel. De igual manera, como técnicas de recolección de información, se utilizaron la primaria y secundaria.

Para llevar a cabo la recolección de la información primaria, se ejecutó un cuestionario enfocado al gerente del hotel, donde las preguntas estuvieron relacionadas con las dimensiones $y$ subdimensiones de la RSE tomando en consideración a la Confederación de la Producción y del Comercio y Prohumana (2006). A su vez, se hizo la respectiva tabulación y se analizaron los datos recolectados y procediendo luego a las gráficas y su respectiva explicación de ellas. Todo lo anterior, sirvió de fundamento para la formulación de las conclusiones y la propuesta de mejora en RSE de la empresa estudiada.

Para la recolección de los datos secundarios, se hicieron revisiones $y$ análisis de bibliografías, tomando en consideración base de datos, páginas web, artículos científicos y tesis que guardan relación a la temática, tanto nacional como internacional. 


\section{RESULTADOS}

En relación a la medición de la RSE utilizado en el Hotel Sonesta de la ciudad de Cartagena, se tomó como referente al documento de la Confederación de la Producción y del Comercio y Prohumana (2006), por medio de ella se pudo promediar cada dimensión analizada.

Por lo tanto, los resultados arrojados por el hotel encuestado en relación a las dimensiones y subdimensiones de la RSE fueron:

En la primera dimensión, Valores y Coherencia, el rango está distribuido de la siguiente manera: Menor hasta 13 puntos es insuficiente; de 14 hasta 21 puntos es regular; de 22 hasta 29 puntos es bueno y, de 30 puntos a más es excelente. El hotel arrojó un puntaje total de 34 puntos, siendo considerado en este punto como una excelente gestión.

A su vez, las subdimensiones presentaron el siguiente resultado: Los mayores puntajes se dieron con Código de Ética con 13 puntos y Gobierno Corporativo con 9 puntos. En un punto intermedio se presentó Reportes de Sustentabilidad con 6 puntos; y entre los menores puntajes, Relaciones Leales con la Competencia con 4 puntos y Transparencia en los Informes Contables con 2 puntos respectivamente.

La siguiente figura, se muestra el análisis de las subdimensiones dadas:

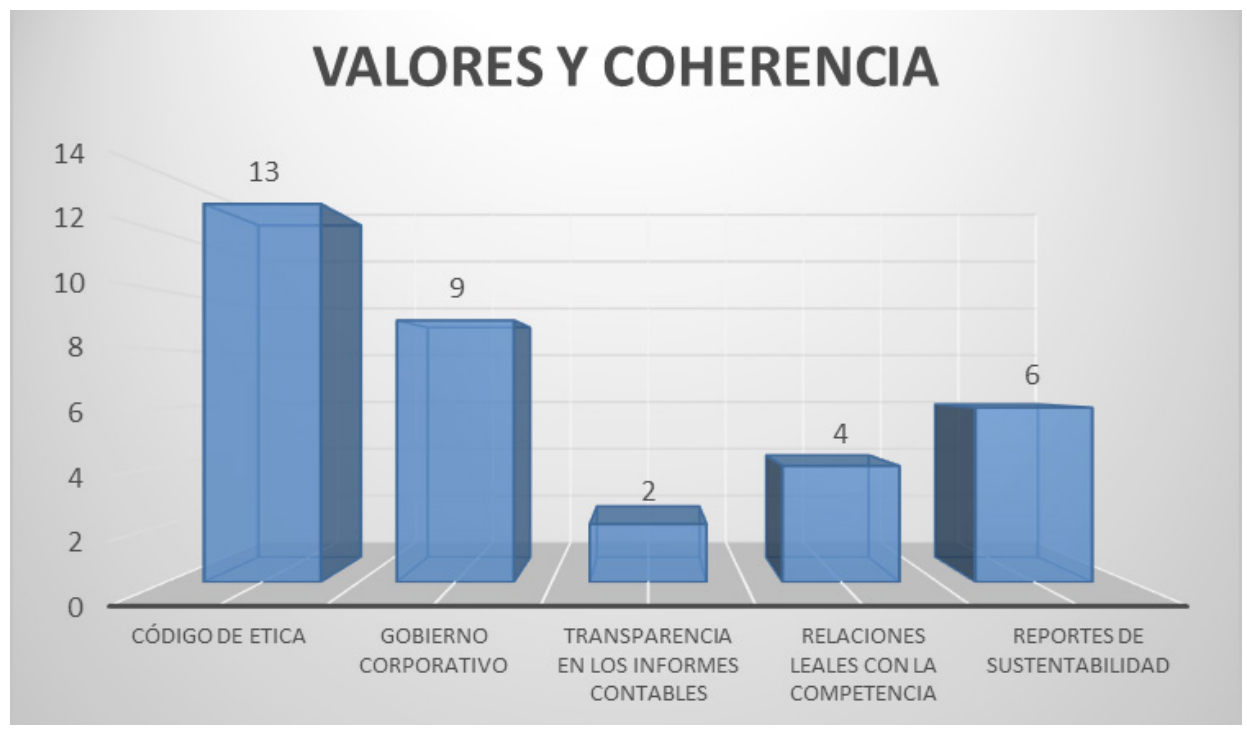

Fuente: Los investigadores

En la segunda dimensión, Público Interno, el rango se encuentra distribuido de la siguiente forma: Menor hasta 22 puntos es insuficiente; de 23 hasta 27 puntos es regular; de
38 hasta 52 puntos es bueno y, de 53 puntos a más es excelente. El hotel tuvo un puntaje total de 65 puntos, por lo que se considera que tiene una excelente gestión corporativa. 


\section{Figura 2. Público Interno}

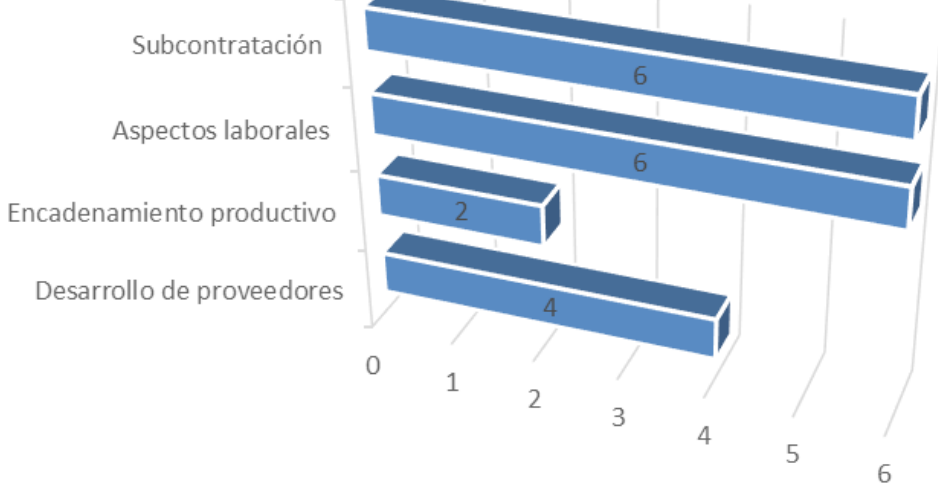

Fuente: Los investigadores

A su vez, las subdimensiones presentaron el siguiente resultado: Los mayores puntajes se dieron en Seguridad e Higiene y otra como la Conciliación Vida Personal y Vida Laboral con 10 puntos cada una de ellas. Le sigue Política de Diversidad con 8 puntos, seguido por Políticas de Beneficios con 7 puntos. Luego, Política de Salud, Políticas de Capacitación y Políticas de Prevención y Sanción del Acoso con 6 puntos cada uno de ellos y, finalmente, con 6 puntos cada uno de ellos, Política con los Sindicatos u Organizaciones de Trabajadores, Políticas de Contratación y Política de Previsión.

La figura 2, se muestra el análisis de las subdimensiones expuestas.

En la tercera dimensión, Relación con los Proveedores, el rango presentado se da de la siguiente manera: Menor hasta 10 puntos es insuficiente; de 11 a 16 puntos es regular; de 17 a 22 puntos es bueno y, de 23 puntos a más es excelente. El hotel Las Américas, logró un puntaje total de 18 puntos, por lo que es considerado como bueno en su gestión empresarial.
Las subdimensiones se dieron de la siguiente forma: Con mayores puntos se encuentran Aspectos Laborales y Subcontratación con 6 puntos cada uno de ellos. Desarrollo de Proveedores con 4 puntos y, con el menor puntaje, Encadenamiento Productivo, con 2 puntos.

La figura 3, se muestra el análisis de las subdimensiones expuestas.

En la cuarta dimensión, Relación con los Consumidores, los resultados en sus subdimensiones fueron: El de mayor puntaje, Relación con los consumidorescon 8 puntos, seguido de Política Publicitaria y Conocimiento de Daños Potenciales con 4 puntos cada uno de ellas.

Los puntajes de esta dimensión se exponen así: Menor y hasta 7 es insuficiente; entre 8 y 12 es regular; entre 13 y 17 es bueno $\mathrm{y}$, de 18 puntos a más, es excelente. Por lo tanto, según los datos recopilados de esta dimensión, la empresa hotelera muestra un resultado total de 16 puntos, por lo que se le puede considerar como bueno, tal como se muestra en la figura 4. 


\section{Figura 3. Relación con los proveedores}

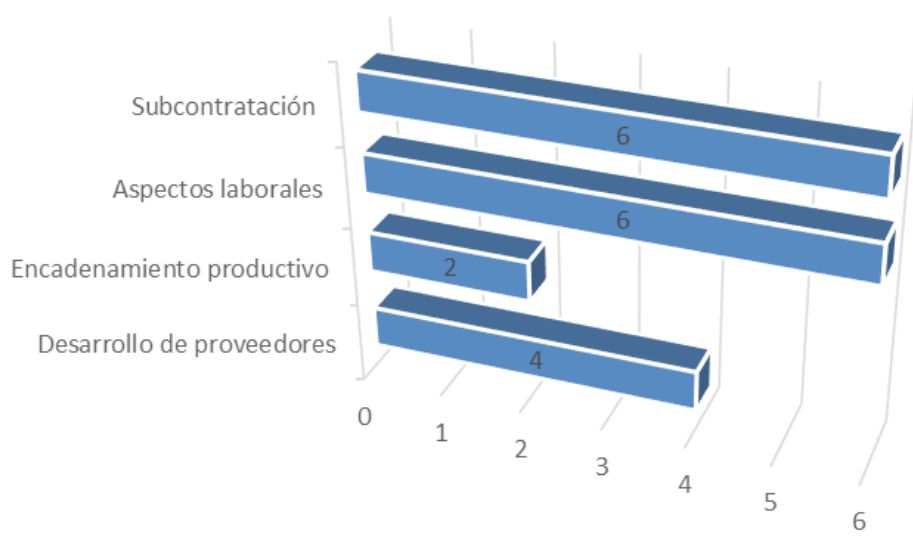

Fuente: Los investigadores

\section{Figura 4. Relación con los Consumidores}

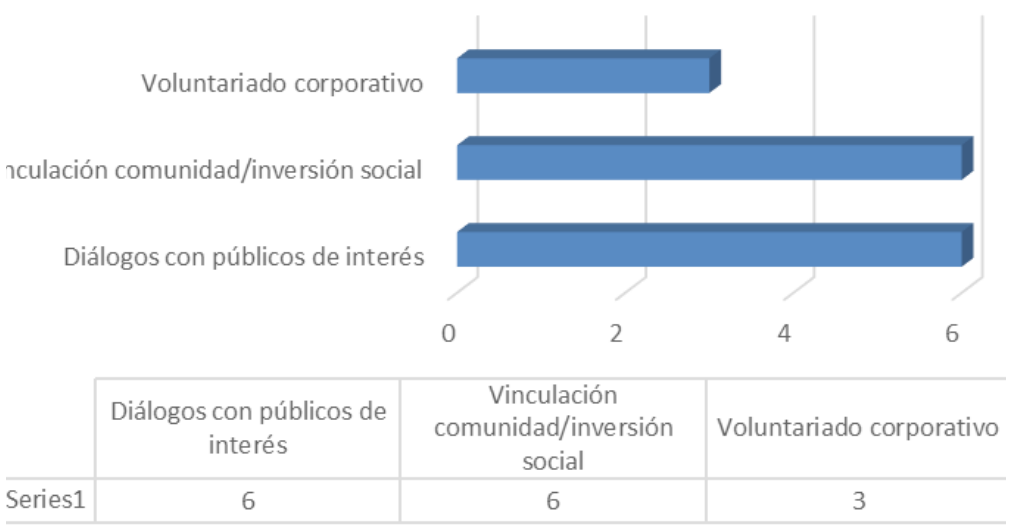

Fuente: Los investigadores

Figura 5. Relación con la comunidad

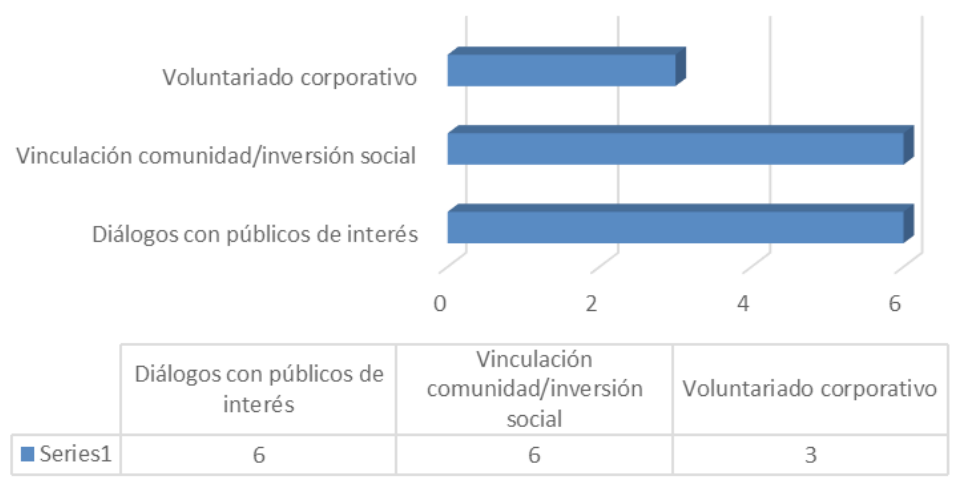

Fuente: Los investigadores 
En la quinta dimensión, la dimensión Relación con la Comunidad, presenta lo siguiente: las subdimensiones con mayor puntaje fueron Diálogos con Públicos de Interés y Vinculación Comunidad/Inversión Social, con 6 puntos respectivamente, seguido de Voluntariado Corporativo, con solamente 3 puntos.

Los puntajes dados por esta dimensión, son: Menor y hasta 7 es insuficiente; entre 8 y 12 es regular; entre13 y 17 es bueno y, de 18 a más, es excelente.

Ante lo expuesto, la corporación hotelera muestra un puntaje de 15, lo cual equivale a una medición buena, tal como se muestra en la siguiente figura.

La sexta y última dimensión llamada Medio Ambiente, presenta en sus subdimensiones la siguiente evaluación: Prácticas Ambientales, la cual presenta el mayor puntaje, siendo este 14. Seguido con 10 puntos, Actitud Proactiva Frente a las Exigencias Medioambientales y, con el menor puntaje, Sistema de Gestión Medioambiental, con solamente 2 puntos.
Los puntajes que presenta esta dimensión se da de la siguiente manera: Menor y hasta 11 es insuficiente; entre 12 y 18 es regular; entre 19 y 25 es bueno y, de 26 a más, es excelente.

Por consiguiente, el hotel muestra un total de 26 puntos, lo cual lo coloca en el rango de excelente, tal como se muestran en la siguiente figura.

\section{CONCLUSIONES}

Al hablar de la responsabilidad social corporativa se evidencia el accionar de una empresa y el cual se encuentra analizado en los enfoques contemporáneos organizacionales, teniendo como fin la incorporación de elementos sociales dentro de la toma de decisiones que ella hace y que repercuten en sus miembros y en su entorno social, la cuales son afectadas de manera positiva o negativa.

Esta investigación muestra de manera genérica que la corporación hotelera objeto de estudio sí se encuentra verdaderamente comprometida con la RSE dentro de su

Figura 6. Medio Ambiente

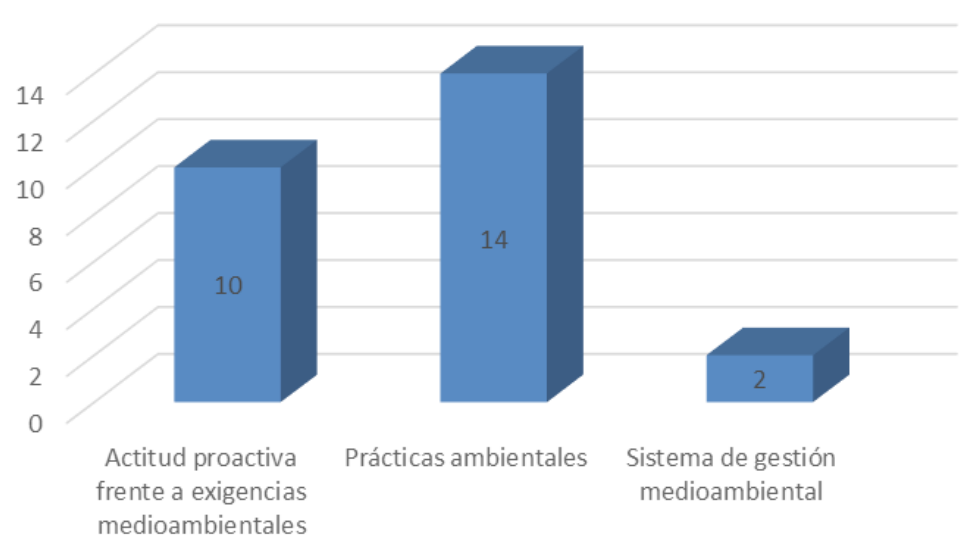

Fuente: Los investigadores 
gestión, esto porque al estudiar las seis dimensiones que forman parte de ella, se obtuvo resultados excelentes en Valores y Coherencia, en Público Interno y en Medio Ambiente. A la vez, Proveedores, Relación con los consumidores y Relación con la Comunidad, obtuvo como resultados buenos; no presentando resultados ni regulares e insuficientes.

Al fin y al cabo, la RSE debe ser una estrategia competitiva que dé a luz beneficios y valor agregado a la empresa; caso contrario, perdería su atractivo en la clase empresarial.

\section{REFERENCIAS BIBLIOGRÁFICAS}

Anturi, R. (2015). El análisis técnico y fundamental en un contexto de globalización: Bancolombia. Aglala, Vol. 6 (1), 1-37. doi:10.22519/22157360.753

Almagro, J. (2011). Aproximación a la responsabilidad social de la empresa: Reflexiones y propuesta de un modelo. España: Fundación Mapfre.

Bateman, T. y Snell, S. (2005). Administración: Un nuevo panorama competitivo. México: McGraw-Hill/Interamericana

Castro, A. (2013). Ética empresarial en la responsabilidad social universitaria. Aglala, Vol.4, (1), 1-13. doi:10.22519/22157360.728

Castro Alfaro, A. (2015). Ética empresarial en la responsabilidad social universitaria. Aglala, 4(1), 1-13. doi:10.22519/22157360.728

Castro, A. (2013). La responsabilidad social universitaria en la formación de una cultura ética en una sociedad globalizada. Global Conference on Business and Finance Proceedings, Vol. 8, 2. 2052-2061

Castro, A. (2014). Planificación territorial en la ciudad de Cartagena: Una relación dialéctica entre desarrollo sostenible y sostenibilidad ambiental. Aglala, Vol. 5, (1), 1-20. doi:10.22519/22157360.698
Castro Alfaro, A. (2014). Planificación territorial en la ciudad de Cartagena: Una relación dialéctica entre desarrollo sostenible y sostenibilidad ambiental. Aglala, 5(1), 1-20. doi:10.22519/22157360.698

Castro, A., Hernández, J. y Bedoya, E. (2016). Caracterización de las dimensiones de la responsabilidad social corporativa en la empresa hotelera Almirante Cartagena. Saber, Ciencia y Libertad, 11,2: 121-132. Recuperado en: http://www. sabercienciaylibertad.org/ojs/index.php/ scyl/article/view/196

Castro, A., Marrugo, G., Gutiérrez, J. y Camacho, Y. (2014). La convivencia y la mediación de conflictos como estrategia pedagógica en la vida escolar. Panorama Económico. Vol. 22, 1. 169-190. Recuperado en: http:// revistas.unicartagena.edu.co/index.php/ panoramaeconomico/article/view/1368

Cifuentes, O. (2012). Aplicación del alineamiento del Supply Chain Management como estrategia competitiva en las empresas del Clúster Logístico de Cartagena. Aglala, Vol. 3, 1, 122-146. doi:10.22519/22157360.348

Cifuentes Cifuentes, O. (2013). Aplicación del Alineamiento del Supply Chain Management como estrategia competitiva en las empresas del Clúster Logístico de Cartagena. Aglala, 3(1), 122-146. doi:10.22519/22157360.348

Cortés, I. (2013). La responsabilidad social empresarial en la economía global. Tesis de Especialización. Universidad Militar Nueva Granada. Facultad de Ciencias Económicas y Administrativas. Bogotá-Colombia

Confederación de la Producción y del Comercio y Prohumana (2006). Manual de RSE para pymes. Recuperado en http://prohumana. cl/documentos/Guia_RSE_NUEVA.pdf

Del Río, J., Cardona, D. y Pérez, I. (2012). Desarrollo de actividades de emprendimiento con innovación y responsabilidad social en los hoteles de la ciudad de Cartagena de Indias. Saber, Ciencia y Libertad, 7: 113-124. Recuperado en: http://sabercienciaylibertad.com/ojs/ index.php/scyl/article/view/130 
Freeman, R. (1984). Strategic management: a stakeholders approach. Boston MA: Pitman

Llano, P. (2014). La flexibilidad laboral y el salario emocional. Aglala, Vol.5, 1, 34-68

Londoño-Aldana, E. (2007). Imagen y posicionamiento de las tiendas de barrio en Colombia aplicando el análisis factorial de correspondencia (AFC). Panorama Económico, 15: 267-293. Recuperado en: http://revistas.unicartagena.edu.co/ index.php/panoramaeconomico/article/ view/407

Maldonado, J. (2015). Análisis de los factores críticos que afectan la competitividad de los puertos para la navegabilidad en el río Magdalena. Aglala, Vol.6, 1, 72-101

Mendoza Ramos, L. (2013). La educación como factor competitivo en Cartagena de Indias D.T y C. Aglala, 3(1), 40-64. doi:10.22519/22157360.345

Mitchel, R. K., Agle, B. R. y Wood, D. J. (1997). Toward a theory of stakeholders identifications and salience: Defining the principle of who and what really counts. Academy of Management Review.

Navas, M. y Londoño, E. (2015). Las fundaciones y su concepción y gestión de la responsabilidad social. Saber, Ciencia y Libertad, 10, 1: 87-100. Recuperado en: http://www.sabercienciaylibertad.org/ojs/ index.php/scyl/article/view/44

Ocampo, M. (2014). Sistema bancario colombiano y la educación financiera. Caso Banco Davivienda. Aglala, Vol.5, 1, 143-167

Quesada-Ibargüen, V. (2003). Estimación de la eficiencia mediante el Análisis Envolvente de Datos (DEA). Panorama Económico, 11: 7-33. Recuperado en: http:// revistas.unicartagena.edu.co/index.php/ panoramaeconomico/article/view/463

Romero Hernández, A. (2010). La planeación tributaria y financiera en la consecución de un mismo fin "maximizar utilidades". Aglala, 1(1), 127-135. doi:10.22519/22157360.717

Ruiz, M. y Romero, Z. (2011). La responsabilidad social empresarial y la obsolescencia programada. Saber, Ciencia y Libertad, 6, 1: 127-135. Recuperado en: http://www. sabercienciaylibertad.org/ojs/index.php/ scyl/article/view/68

Server, R. y Capo, J. (2011). The interrelationship between the demands of Corporate Social Responsability and co-operative principles and values. Revista de Economía Pública, Social y Cooperativa, 73. 213-232

Serna, F.; Barrera, L. y Montiel, H. (2011). Impacto social y económico en el uso de biocombustible. Journal of Technology Management and Innovation, 91-97.

Vergara-Schmalbach, L., Amezquita, López, J., \& Maza-Avila, F. (2008). Diseño y análisis de escenarios a parir de la caracterización de las cadenas productivas mediante modelos de redes. Panorama Económico, 16: 73-89. Recuperado en: http://revistas. unicartagena.edu.co/index.php/ panoramaeconomico/article/view/379

Vergara, J. y Carbal, A. (2014). Diseño de un sistema de gestión en responsabilidad social empresarial para pequeños hoteles de la ciudad de Cartagena. Saber, Ciencia y Libertad, 9, 2: 91-108. Recuperado en: http:// www.sabercienciaylibertad.org/ojs/index. $\mathrm{php} / \mathrm{scyl} / \mathrm{article} / \mathrm{view} / 7$

Vives, A. y Peinado-Vara, E. (2011). Manual de gestión, la responsabilidad social empresarial en América Latina. Recuperado en: https://www.pwc.com/cl/es/publicaciones/ assets/la-responsabilidad-social-de-la-empresa-en-america-latina.pdf

Castro-Alfaro, A., Mercado-León, L., \& GonzálezPérez, N. (2016). El capital humano como factor de competitividad en la industria hotelera y turística en Cartagena. Panorama Económico, 24, 283-296. 


\section{AUTORES}

Alain Castro-Alfaro

Sociólogo de la Universidad Inca Garcilaso de la Vega (Perú). Magister en Gestión de la Alta Dirección de la Universidad Nacional Federico Villareal (Perú). Director de la empresa de asesorías en investigación científica Tesis Colombia. Docente Investigador de la Corporación Universitaria Rafael Núñez. Miembro del Grupo de Investigación Cartaciencia.

Lino Mercado-León

Magíster en Dirección de Marketing de la Universidad Viña del Mar (Chile). Especialista en Gerencia de Mercadeo de la Universidad Tecnológica de Bolívar (Colombia). Administrador de Empresas de la Universidad de Pamplona (Colombia). Actualmente es Docente Universitario de la Fundación Tecnologico Comfenalco en el Programa de Ingeniería Industrial, Catedrático Universidad de Cartagena adscrito a los programas de Administración de Empresas y Administración Industrial. Alterna el el ejercicio de la Docencia con la Consultoría y Conferencias en el área de Marketing, Branding y Management.

Nora González-Pérez

Estudiante del programa de Contaduría Pública de la Universidad IAFIT (Colombia). 